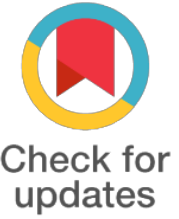

*For correspondence:

ngdluyen@hotmail.com

Competing interests: The authors declare that no competing interests exist.

Received: 2017-07-15

Accepted: 2017-08-05

Published: 2017-09-05

Copyright The Author(s) 2017. This article is published with open access by BioMedPress (BMP).

This article is distributed under the terms of the Creative Commons Attribution License (CC-BY 4.0) which permits any use, distribution, and reproduction in any medium, provided the original author(s) and the source are credited.
POSTER

\section{Synthesis and biological evaluation of novel curcumin derivative with water-soluble phosphate group as potential antitumor agent}

\author{
Dinh Luyen Nguyen, Van Giang Nguyen, Thi Bich Van Nong, Thi Hien \\ Pham, Van Hai Nguyen
}

Department of pharmaceutical industry, Hanoi University of Pharmacy, Hanoi, Hanoi 84, Vietnam

\section{Abstract}

Background: Curcumin (diferuloylmethane) is the main natural active polyphenol from the rhizome of Curcuma longa (turmeric) and in others Curcuma spp. The main pharmacological effects include anti-tumor, anti-inflammatory, anti-oxidation, anti-fungal and anti-bacterial activities. However, the clinical application of curcumin is limited by its main drawbacks such as low solubility, poor bioavailability and rapid excretion from the body. In this study, the chemical modification approach was performed to improve the solubility of curcumin in water, at the same time increase the anti-tumor activity.

Methods: The water-soluble compound (2-(curcumin-O-yl)ethyl dihydrophosphate, LH-42017) was synthesized by two classical reactions: O-alkylation with 2-bromoethanol to obtain monoO-(2-hydroxyethyl)-curcumin, followed by esterification using phosphoryl chloride in water and pyridine. The chemical structure was confirmed by IR-, NMR-spectroscopies and massspectrometry. The anti-tumor activity of $\mathrm{LH}-42017$ was assessed against hepatocellular carcinoma Hep-G2, human breast cancer MCF-7, human leukemia $\mathrm{K}_{5} 62$ and human cervical carcinoma HeLa cell lines based on the sulforhodamine B (SRB) assay.

Results: LH-42017 was successfully synthesized from curcumin by overall yield of $28.43 \%$. IC 50 of original curcumin was 63.55, 90.88, 104.99 and 60.24 $\mathrm{MM}$ on Hep-G2, MCF-7, K562 and Hela, respectively. IC 50 of LH-42017 was $36.82,51.60,60.80$ and $26.14 \mu \mathrm{M}$ on Hep-G2, MCF-7, $\mathrm{K}_{5} 62$ and Hela, respectively.

Conclusion: The novel synthesized compound LH-42017 showed the antitumor activity in 1.7-2.3 times better than original curcumin. The water-soluble phosphate group plays an important role in development of natural curcumin as potential antitumor agent.

\section{Keywords}

Curcumin, hydroxyethyl, phosphate, water-soluble

Funding

References 